\title{
The Warm Spitzer Mission For The Investigation Of Nearby Galaxies
}

\author{
D. Calzetti*, M. Regan ${ }^{\dagger}$, L. van Zee**, L. Armus ${ }^{\ddagger}$, R. Chandar ${ }^{\S}$, K.D. \\ Gordon ${ }^{\mathbb{I}}$, K. Sheth* and M. Yun* \\ *Department of Astronomy, University of Massachusetts, 710 N. Pleasant St., Amherst, MA 01003, \\ USA \\ ${ }^{\dagger}$ Space Telescope Science Institute, 3700 San Martin Dr., Baltimore, MD 21218, USA \\ ${ }^{* *}$ Department of Astronomy, Indiana University, 727 E. 3rd St., Bloomington, IN 47405, USA \\ ${ }^{*}$ Spitzer Science Center, Caltech MC 220-6, Pasadena, CA 91125, USA \\ ${ }^{\S}$ Carnegie Observatories, 813 Santa Barbara St. Pasadena, CA 91101, USA \\ ISteward Observatory, University of Arizona, 933 N. Cherry Ave., Tucson, AZ 85721, USA
}

\begin{abstract}
Spitzer in the warm phase will provide a unique opportunity for large-field and deep observations of nearby galaxies to address a number of fundamental science questions that cannot be adequately answered within the confines of current sample sizes/depths as produced by regular general observer, or even Legacy, proposals. Potential science goals include the morphological classification of galaxies, the investigation of the edges of galaxy disks and of intragroup/intracluster environments, the use of hot dust emission to trace star formation and AGNs, the monitoring of supernovae and of other variable sources, and the calibration of stellar population models in the mid-IR. The local Universe is the only available benchmark against which distant galaxies can be compared, and every effort should be made to ensure that the properties of nearby galaxies are fully investigated by the 'warm' Spitzer.
\end{abstract}

Keywords: Spitzer Space Telescope, infrared astronomical observations, external galaxies, galaxy formation, stellar populations

PACS: 95.85.Hp, 98.62.Ai, 98.62.Ck, 98.62.Lv

\section{INTRODUCTION}

Spitzer in the warm phase will provide a unique opportunity for large-field and deep observations of nearby galaxies, to address a number of fundamental science questions that cannot be adequately answered within the confines of current sample sizes/depths as produced by regular general observer, or even Legacy, proposals.

The science goals, which will be detailed in the next few sections, include the morphological classification of galaxies, the investigation of the edges of galaxy disks and of intragroup/intracluster environments, the use of hot dust emission to trace star formation and AGNs, the monitoring of supernovae (for the study of dust production) and of other variable sources, and the calibration of stellar population models in the mid-IR.

The exploration of the nearby Universe is not only important per se, but it also provides the ultimate benchmark for unraveling the distant Universe. Hence, the utilization of the 'Warm' Spitzer for the investigation of the properties of nearby galaxies will have the double benefit of providing for the understanding of both the local Universe and the distant galaxies populations.

These goals can be accomplished with a combination of both Super-Legacy Surveys

$$
\begin{aligned}
& \text { CP943, The Science Opportunities for the Warm Spitzer Mission Workshop, } \\
& \text { edited by L. J. Storrie-Lombardi and N. A. Silbermann } \\
& \text { (C) } 2007 \text { American Institute of Physics } 978-0-7354-0457-1 / 07 / \$ 23.00
\end{aligned}
$$


and targeted observations. Independent of the nature of the observing project, the science goals described below have the potential to generate data that both provide an enduring archival research value and are pathfinders or foundation science for existing or upcoming facilities, both at infrared/submillimeter wavelengths (e.g., Herschel, JWST, etc.), and $\mathrm{mm}$ /radio wavelengths (ALMA, CARMA, LMT, EVLA, etc.). As an example, by the time ALMA is operational at full capacity in 2012, JWST will not yet be in space, and other NASA Great Observatories need to provide the critical complementary, multiwavelength data while they are still operating.

IRAC on the Warm Spitzer will be the most sensitive camera available at 3.6 and $4.5 \mu \mathrm{m}$ for such applications until the advent of JWST. IRAC still has one advantage over JWST, in that its field of view is 2.6 times larger, a crucial feature for observations of extended galaxies. IRAC on the Warm Spitzer will also be about twice as sensitive than WISE, and with a much higher angular resolution $(\sim 2 \operatorname{arcsec}$ for IRAC versus $\sim 6$ arcsec for WISE). These capabilities are used in what follows as guidance to identify the unique science contributions that the Warm Spitzer mission can provide in the field of nearby galaxies studies.

\section{SCIENCE DRIVERS}

\subsection{Stellar Masses And The Morphological Classification Of Galaxies}

The stellar mass and the morphology of a galaxy are two of the primary tools to investigate galaxy evolution. Galaxies are routinely decomposed into their primary stellar components: bulge, disk, bars (and/or into other objective elements, such as concentration parameters, second order moments, asymmetry parameters, and Gini coefficients (Bershady et al. [1], Conselice [2], Lotz et al. [3]), to map their history across cosmological times.

At optical wavelengths, stellar masses and morphological classifications have been historically hampered or complicated by the presence of dust, and by the sensitivity of some of the indices to presence of active regions of star formation (which tend to make some features more prominent than others, without necessarily tracing the underlying stellar mass). At infrared wavelengths, the impact of dust extinction and of localized star formation decreases by at least one order of magnitude. At these wavelengths, spiral structures become less evident, and bulges and bars become more prominent.

Tracing the underlying mass and structure of galaxies requires statistically large samples of nearby galaxies observed at infrared wavelengths. Because of their proximity, nearby galaxies are the natural test-beds for extant and new classification schemes, thanks to the higher spatial resolution they provide relative to their more distant counterparts.

2MASS (Skrutskie et al. [4]) has provided a first 'cut' to a sample like the one proposed here, but far from optimal in terms of depth. The typical 2MASS galaxy has been observed with a depth that is about 1-2 orders of magnitude shallower than the surface brightness at the $\mathrm{R} 25$ isophotal radius. This isophotal radius is the canonical value used in most classical morphological surveys. Deep infrared observations from the ground are far less efficient than from space, due to the many hundred-fold increase in the back- 
ground. Furthermore, observations in the $\mathrm{K}$ band suffer from larger contamination from red supergiants (which trace recent star formation) than longer wavelengths.

A 'Spitzer RC3' would be the ultimate survey of this kind, but a unbiased sample selected from existing catalogs of about 1,000-1,500 targets (about 150-200 galaxies per morphological bin) would still accomplish much of the science goals above. This is the type of project, both in terms of required observing and data products uniformity and of sheer amount of observing time, that would easily lend itself to be SSC-led as a service to the community.

\subsection{The Edges Of Disks: Pathfinders Of Galaxy Evolution}

A number of studies, mostly based on HST optical and GALEX UV imaging, have revealed the presence of low surface brightness features at very extended radii associated with most galaxies. GALEX has shown that in a number of nearby galaxies the UV emission extends well beyond the 'edge' of the H-alpha disk, and beyond 2-3 times the Holmberg radius (Thilker et al. [5], Gil de Paz et al. [6]). This UV emission is due to stellar populations, for the most part 'evolved' (i.e., no longer producing ionizing photons, but still young enough to produce significant UV, i.e. $10 \mathrm{Myr}<$ ages < a few hundred Myr; Dong et al. 2007, in prep.). Longer wavelength observations have further shown the presence of stellar populations older than a few hundred Myr beyond the standard edge of the disks. Clearly, there is and/or has been star formation in these extreme environments, possibly with multiple episodes or complex histories.

Presence of extended disks, and the presence or absence of an edge to the stellar population, have profound implications for our understanding of the formation and evolution of disks. The extreme questions one may be tempted to ask are: Where does one galaxy end and another begin? Are all galaxies just one humongous galaxy? Optimal targets for this type of investigation are inclined galaxies, which maximize the contrast against the background.

Not less important are the implications of current or recent-past star formation for our understanding of the physics of the scaling laws of star formation and of local versus global star formation thresholds (in a potentially low-gas-density environment like the outer disk regions). Outer-disk star formation has also the potential to play a crucial role for the pollution of the intergalactic medium, which would be much more easily done from the 'edges' of the potential wells. Examples of targets include the galaxies for which GALEX has detected extended (beyond the disks) UV emission.

Along the same lines, the intragroup and intracluster environments provide excellent laboratories for observing galaxy evolution in action, including harrassment, ram pressure stripping, and tidal encounters. Tidal tails or accretion driven events may produce gaseous features that last a significant period of time $(\sim 1 \mathrm{Gyr})$. These tidal features are attractive for the investigation of the local star formation threshold, as they provide a high-density environment far away from the inner disks of galaxies. Blind surveys of nearby groups and clusters will enable identification of so-far unknown faint galaxies (red and dead) and the investigation of tidal streams and intragroup/intracluster old stellar populations. An example is a blind survey of all of the Virgo Cluster, or at least the 
inner 20deg x 20deg portion. Virgo is both the nearest large cluster and is non-virialized, implying that galaxies in a range of environments are present.

Deep Spitzer observations at 3.6 and $4.5 \mu \mathrm{m}$ would target the evolved stellar populations at the edges of galaxies and/or in the intragroup/cluster medium, thus providing a more direct link than shorter wavelength observations to the mass of the stellar populations formed in these environments. As already remarked in the previous section, the 3.6 and $4.5 \mu \mathrm{m}$ bands have lower contamination from red supergiants than the ground-based $\mathrm{K}$ band, and are therefore much more efficient tracers of the old, evolved stellar populations. The full census of these populations provided by Spitzer, together with existing supporting observations (e.g., HI deep maps, UV images, etc.), will shed light on their nature, and address questions on the formation and evolution of their parent galaxies.

\subsection{Hot Dust And Star Formation}

The investigation of the SINGS galaxies sample (Kennicutt et al. [7]) shows that the ratio 4.5/3.6 varies significantly across galaxy disks, and it correlates strongly with tracers of star formation, like the IRAC $8 \mu \mathrm{m}$ emission, in the sense that higher $8 \mu \mathrm{m}$ emission corresponds to large values of the 4.5/3.6 ratio. The interpretation is that the recently formed stars heat the dust to temperatures high enough to contribute to the emission in the 4.5 IRAC band.

Further investigations are needed to provide a full characterization of the 4.5/3.6 ratio as a SFR indicator, and if a reliable calibration can be derived, this ratio would provide a potentially interesting tracer of star formation because of its insensitivity to extinction. However, existing 3.6 and 4.5 images are generally not deep enough to probe the full dynamical range of the ratio between the two bands.

Deep 3.6 and 4.5 IRAC images of nearby galaxies would provide the needed characterization (e.g., by comparison with archival MIPS $24 \mu \mathrm{m}$ images, which are more closely related to current SFR, Calzetti et al. [8]), and, subsequently, a potentially powerful application for the mapping of SFRs in nearby galaxies. This would complement efforts to map the evolved stellar populations (see below) and stellar masses.

\subsection{Hot Dust And AGNs}

A common infrared spectral signature of Active Galactic Nuclei (AGN) is an excess of very hot $(\mathrm{T}>500 \mathrm{~K})$ dust emission over a stellar continuum. This dust is thought to exist at the inner edge of a torus, or on the nuclear-facing surfaces of individual clouds, at small radii $(\mathrm{r}<50 \mathrm{pc})$, directly illuminated by the AGN accretion disk. The dust produces strong blackbody emission throughout the mid-infrared, and in some cases, a 'bump' in the spectrum at $10 \mu \mathrm{m}$ from silicate emission. In sources with powerful AGN, or a lot of obscuring dust near the nucleus, the hot dust continuum can be detected over a very large range in wavelengths, extending into the near-infrared and far-infrared parts of the spectrum. In fact, the $25 / 60 \mu \mathrm{m}$ flux density ratio was used very effectively over 20 years ago to isolate active galaxies in the IRAS database (De Grijp et al. [9], Miley 
et al. [10]).

The IRAC 3.6 and $4.5 \mu \mathrm{m}$ bands are very sensitive to this hot dust emission, and AGN can be separated from star-forming galaxies in large Spitzer surveys (e.g. FLS, SWIRE, GOODS) using IRAC alone (Lacy et al. [11], Stern et al. [12]). While all four IRAC bands are typically used to constrain the mid-infrared spectral energy distribution, the 3.6 and $4.5 \mu \mathrm{m}$ bands provide most of the power in detecting the excess emission from the hottest dust, over that from stars, since the stellar light is dropping while the hot dust is climbing.

A warm mission science program combining near-infrared $(\mathrm{J}, \mathrm{H}, \mathrm{K})$ data with 3.6 and $4.5 \mu \mathrm{m}$ IRAC band data would effectively isolate AGN for redshifts $\mathrm{z}<1$. At these redshifts the ground-based $\mathrm{K}$ and $\mathrm{L}$ bands move through the 3.6 and 4.5 IRAC bands. For $\mathrm{z}>1$, stellar emission dominates those bands, making detection of hot dust extremely difficult, except for the most luminous AGN (whose near-infrared light shortward of the $\mathrm{K}$-band can still be dominated by dust emission). Starburst galaxies tend to get redder in their IRAC colors for $\mathrm{z}>1$. AGN typically have $\mathrm{m}_{3.6}-\mathrm{m}_{4.5}=0.5-1.0 \mathrm{mag}$ (Vega), while starburst galaxies tend to be $0.5-1.0$ mag bluer for $\mathrm{z}<0.3$ and $0.5<\mathrm{z}<1.0$ (Stern et al. 2005).

A program that targets $\mathrm{AGN}$ at $\mathrm{z}<1$, in order to quantify the amount of hot dust, and compares this to the optical emission line (e.g. [OIII]) luminosity or the radio power, would be useful for modeling the properties of the torus or obscuring clouds. Samples could be built from SDSS, FIRST, or other surveys that focus on properties not directly associated with near-nuclear emitting dust. A careful accounting of the detection limits (as a function of AGN-to-host luminosity) would be required in order to properly assess the statistics, since the IRAC observations would have the largest contribution from host (stellar) light. However, the IRAC data would be extremely sensitive to classes of (moderately) obscured AGN, such as those uncovered in the 2MASS survey (Cutri et al. [13]), which are not readily found via more traditional means. The sensitivity of IRAC coupled with the mapping speed of Spitzer makes it possible to build up complete samples of AGN, extremely rapidly. Very large, near-infrared based surveys (e.g., UKIDSS) could take full advantage of pointed or mapped IRAC follow-up, even with only the 3.6 and 4.5 bands. The planned availability of extremely large or all sky surveys in the optical and mid-infrared (e.g., LSST, WISE) when combined with IRAC 3.6 and $4.5 \mu \mathrm{m}$ data, would provide an extremely valuable tool for both finding hidden AGN, and understanding accretion process, by tying optical or UV variability to the response of the (reprocessed) dust emission. Even if a focused survey for buried AGN is not done, a complete assessment of the number density of galaxies hosting obscured AGN, as revealed through their excess hot dust emission, can arguably be a key science driver for other extragalactic surveys being planned for the warm mission.

\subsection{A Census Of Evolved Stellar Populations: The Local Group}

The proximity of Local Group Galaxies offers the unique opportunity to map their evolved stellar populations with a spatial resolution that is unmatched by more distant galaxies. This can provide a complete census of such populations within a well defined 
volume of the Universe, and complement similar observations at other wavelengths, to investigate the evolution of Local Group Galaxies.

During the Warm Spitzer mission, an investigation of the archive should be performed to establish which Local Group galaxies have been observed (down to R25), which need supplementary observations, and which are missing altogether and thus need observing. The complete local volume observed at 3.6 and $4.5 \mu \mathrm{m}$ will provide an enduring legacy for future observations, and lends itself to also be SSC-led, and be provided as a service to the community.

As an example of scientific application for such observations, we mention the investigation of the dust versus stellar emission in Local Group Galaxies. Although the 3.6 and $4.5 \mu \mathrm{m}$ IRAC bands are contributed mainly by photospheric emission from stars (at least when observing external galaxies), both bands receive contribution from dust as well: aromatic emission at $3.6 \mu \mathrm{m}$ and small grain continuum emission at $4.5 \mu \mathrm{m}$. The proximity of the Local Group Galaxies will provide the opportunity to discriminate the contribution from dust from that of stars, and the two dust contributions as well. The spatial decomposition between stars and dust will help understand how much of the integrated emission in more distant galaxies in these two bands is contributed by dust emission versus stellar emission.

\subsection{Variability Monitoring In Local Group Galaxies}

Spitzer observations have been instrumental in establishing that supernovae may be more efficient at destroying dust than producing it, both for Type II and Type 1a (Borkowski et al. [14], Williams et al. [15]). However, the issue is not entirely settled yet, and some evidence for dust production has been reported, also using Spitzer data (Sugerman et al. [16], Smith et al. [17]). Whether massive-star supernovae are efficient dust factories or not has profound implications for our understanding of the primordial Universe, in particular for our understanding of the dust detected in quasars and GRBs at epochs when the Universe was less than 1 Gyr old (Bianchi et al. [18]). During the warm phase, Spitzer could periodically monitor nearby galaxies for which data already exist, to observe supernovae explosions. Observations at 3.6 and $4.5 \mu \mathrm{m}$ of such events within a short period of their happening will provide insights into the evolution of the warm dust emission (warm enough to emit in the short IRAC bands) from Supernovae.

Recent investigations (e.g., with the SAGE Spitzer data of the LMC) are beginning to explore the connection between stellar variability and mass loss in Local Group galaxies. One idea that is being pursued is that mass loss in stars is driven by variability. Mass loss from stars is traced via IRAC 8 and MIPS $24 \mu \mathrm{m}$, which are good indicators of dust in the winds of stars which are injecting dust+gas into the ISM. Variability could be traced by multiple-epochs observations of those stars at the short IRAC wavelengths, over multiple years during the warm phase. This could be done not only for the LMC, but also for other Local Group galaxies for which suitable data for tracing the dust emitted from stars exist. Indeed, IRAC and MIPS data already exist for most Local Group galaxies, which can be used to trace the mass loss. Even at the distances of M31 and M33, IRAC 3.6 and $4.5 \mu \mathrm{m}$ observations would probe deep enough that we should see a significant fraction of the 
total mass injecting stars. Thus, Spitzer in the warm phase would provide an excellent tool to monitor the variability of the stars for which mass loss has been detected.

\subsection{The Infrared Spectral Energy Distributions Of Stellar Populations}

Some of the science that Spitzer will be able to accomplish during the warm phase could be labeled under the term 'foundation science' for other facilities. Within this category is a Spitzer survey of star clusters covering ages from a few tens of millions of years to 12 billion years and a range of metallicity in nearby galaxies. These observations will provide empirical measurements of simple stellar populations which will be used to improve population synthesis model predictions in the mid-IR (as is currently being done in the near-IR). Such a survey would also enable a direct calibration between metallicity and color in the IRAC bands. In addition, the Spitzer data can be combined with 2MASS observations to create color-magnitude and color-color diagrams of the infrared bright RGB and AGB stars, in order to quantify the relative contributions from the stars and dust as a function of metallicity. These observations will provide benchmarks for interpreting the colors of galaxies and star forming regions within galaxies in the midIR (at both high and low redshift) during the JWST era.

\section{CONTRIBUTION OF WARM SPITZER DATA}

The premise for the science goals described in the previous section is to leverage on the unique capabilities that the Warm Spitzer mission can offer in comparison with other facilities available roughly within the same timeframe and operating at similar wavelengths. Relative to WISE, Spitzer has higher angular resolution ( 2 arcsec versus 6 arcsec for WISE at similar wavelength) and a 30 second IRAC exposure is about twice as sensitive as 8 passes with WISE. The higher angular resolution is a critical requirement for discriminating as much as possible various components in the crowded fields that characterize galaxy observations.

Relative to ground-based near infrared facilities, Spitzer offers a few hundred times lower background and a time-stable PSF (no seeing). These two characteristics are required to probe deeply within and outside galaxies; the lower background from space is a requirement, for instance, for investigating the outer fields of galaxies or the intracluster/intergalactic medium. The superior sensitivity of Spitzer in the 3-5 $\mu \mathrm{m}$ window relative to ground-based instrumentation is also instrumental for reaching the sensitivity limits required for identifying and discriminating AGNs in galaxies via their hot dust emission.

Relative to telescopes with adaptive optics, Spitzer has larger Field-of-View (required for mapping extended objects, like nearby galaxies), stable, repeatable, and uniform PSF, and smaller and stable PSF wings (which enable accurate photometry). Furthermore, the efficient mapping capability is essential for obtaining large maps of nearby objects. 


\section{SCOPE OF DATA}

Each of the science goals described above requires its own datasets, although some observations may address more than one goal.

For goal \#1 (\$2.1), a SINGS-like approach can be adopted, which will require between 1 and 3 hours per galaxy (depending on the size of the galaxy, as the total exposure time per pointing will be fixed at 240 seconds to reach R25). For about 1,500 targets, this translates into approximately 1,500-3,000 hours of observing time.

About 10 times deeper images would be required for characterizing and calibrating the 4.5/3.6 ratio as a SFR tracer, but the number of galaxies required for such investigation would be much smaller, of order of a few tens.

For the outer regions of galaxies and the intragroup/intracluster medium, the goal will be to observe at least small, evolved stellar clusters (masses around 5,000 solar masses and ages about $100-300 \mathrm{Myr}$ ). This would correspond to the detection of 1 microJy at $3.6 \mu \mathrm{m}$, which can be obtained with 2,000 seconds of exposure to achieve $\mathrm{S} / \mathrm{N}=5$. For a $20 \mathrm{deg}$ x $20 \mathrm{deg}$ map (i.e., the inner region of the Virgo Cluster), this would correspond to a total integration time of about 80,000 hours (or about the entire observing time available during the Warm Spitzer mission). Shallower depths (and shorter times) will still yield considerable information on the Virgo structure and the intracluster medium. The outer regions of galaxies will require considerably less total time, because smaller areas will be required to be mapped (although the exposure time per pointing will be roughly the same). In the local Unverse, about $50-100$ targets will have the characteristics required to address science goal \#2 (\$2.2).

Observing time requirements for Local Group Galaxies will be dependent on how many of the galaxies will already be available in the archive by the end of the Cold Spitzer Mission. Exposure times will be also dependent on the extent of the galaxy; as example, a galaxy as extended as the LMC will require a few hundred hours, but a galaxy of similar size as NGC6822 will only require 2 hours.

Variability studies can be used to augment the observations of Local Group Galaxies, by progressively accumulating maps that are observed with a cadence of about $30-60$ days over $5-10$ epochs.

Finally, observations of stellar clusters in external galaxies (goal \#7, §2.7) will have exposure time requirements per pointing that are similar to those of goal \#2 (\$2.2). Optimal targets will be galaxies within 5-10 Mpc that host stellar clusters with a large range of ages and masses within relatively small regions, which can help reduce requirements for mapping. Technically, one could use the same galaxies of goal \#3 (\$2.3) for goal \# 7 $(\$ 2.7)$.

\section{ACKNOWLEDGMENTS}

The authors would like to heartfully thank Nancy Silbermann (Spitzer Science Center) for her extensive help with this manuscript. 


\section{REFERENCES}

1. Bershady, M.A., Jangren, A., and Conselice, C.J. 2000, AJ, 119, 2645

2. Conselice, C.J. 2003, ApJS, 147, 1

3. Lotz, J.M., Primack, J., and Madau, P. 2004, AJ, 128, 163

4. Skrutskie, M.F. 2006, AJ, 131, 1163

5. Thilker, D.A., Bianchi, L., Boissier, S., Gil de Paz, A., Madore, B.F. et al., 2005, ApJ, 619, L79

6. Gil de Paz, A., Madore, B., Boissier, S., Thilker, D., Bianchi, L., et al.,. 2007, ApJ, 661, 115

7. Kennicutt, R.C., Armus, L., Bendo, G., Calzetti, D., Dale, D.A., Draine, B.T., Engelbracht, C.W., Gordon, K.D., Grauer, A.D., Helou, G., et al., 2003, PASP, 115, 928

8. Calzetti, D., Kennicutt, R.C., Engelbracht, C.W., Leitherer, C., Draine, B.T. et al., 2007, ApJ, 666, 870

9. De Grijp, M.H.K., Miley, G.K., Lub, J., and de Jong, T. 1985, Nature, 314, 240

10. Miley, G.K., Neugebauer, G., and Soifer, B.T. 1985, ApJ, 293, L11

11. Lacy, M., Storrie-Lombardi, L.J., Sajina, A., Appleton, P.N., Armus, L., et al., 2004, ApJS, 154, 166

12. Stern, D., Eisenhardt, P., Gorjian, V., Kochanek, C.S., Caldwell, N. et al., 2005, ApJ, 631, 163

13. Cutri, R.M., Nelson, B.O., Francis, P.J., and Smith, P.S., 2002, "The 2MASS Red AGN Survey" in AGN Surveys, ASP Conference Proceedings 284, Astronomical Society of the Pacific, San Francisco, 2002, p. 127.

14. Borkowski, K.J., Williams, B.J., Reynolds, S.P. Blair, W.P., Ghavamian, P. et al., 2006, ApJ, 642, L141

15. Williams, B.J., Borkowski, K.J., Reynolds, S.P., Blair, W.P., Ghavamian, P. et al., 2006, ApJ, 652, L33

16. Sugerman, B.E.K., Ercolano, B., Barlow, M.J., Tielens, A.G.G.M., Clayton, G.C. et al.. 2006, Sci, 313, 196

17. Smith, N., Foley, R., and Filippenko, A.V. 2007, ApJ, submitted (astroph/0704.2249)

18. Bianchi, S., and Schneider, R. 2007, MNRAS, 378, 973 